\title{
Novel expression of VCAM-1 on the mitral valve in a patient with primary antiphospholipid antibody syndrome
}

\author{
M Suguta, Y Hoshino, S Naito
}

A 48 year old Japanese man was admitted to our institute with orthopnoea. $\mathrm{He}$ had undergone coronary angioplasty for acute myocardial infarction six months before and had not had a follow up since that time. A chest radiograph showed severe pulmonary vascular congestion. No significant coronary lesions were seen on coronary angiography. Detailed transoesophageal echocardiography revealed a thickened mitral leaflet with poor coaptation with severe regurgitation, and a dilated left atrium (Fig 1A). Left ventricular function was preserved (ejection fraction $50 \%$ ). Laboratory testing revealed thrombocytopenia and the presence of serum lupus anticoagulant with no evidence of a definite underlying immunologic disorder. The protein $\mathrm{C}$ and protein $\mathrm{S}$ concentrations were normal. Therefore, the patient was diagnosed with primary antiphospholipid

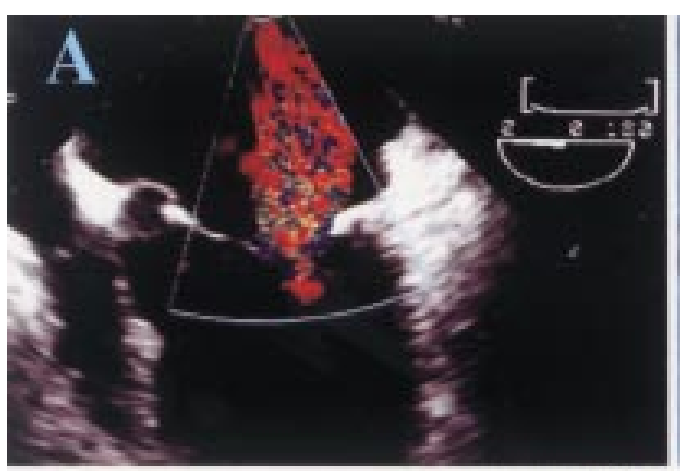

The Second

Department of Internal Medicine, Gunma University School of Medicine, 3-39-15 Showamachi, Maebashi, Gunma 371-8511, Japan M Suguta

Y Hoshino

Gunma Prefectural, Cardiovascular Center, Gunma S Naito

Correspondence to: Dr Suguta Fast5150@aol.com antibody against VCAM-1 (arrow, brown reaction product). syndrome (APS). After aggressive anticoagulation treatment with intravenous heparin, the diseased mitral valve was successfully replaced with a porcine bioprosthesis, and oral warfarin was initiated with a target international normalised ratio of 2.5 to 3 . The patient has done well during 18 months of follow up.

Diffuse thickening of both leaflets of resected mitral valve was noted (fig 1B). Examination of the resected posterior leaflet revealed thickened and oedematous leaflet tissue associated with a perivascular mononuclear infiltrate (fig 1D). Immunostaining revealed VCAM-1 expression around the vasculature (fig $1 \mathrm{E}$ ). These findings suggested that the mononuclear infiltrate and VCAM-1 expression may have played an important role in the pathogenesis of mitral regurgitation.
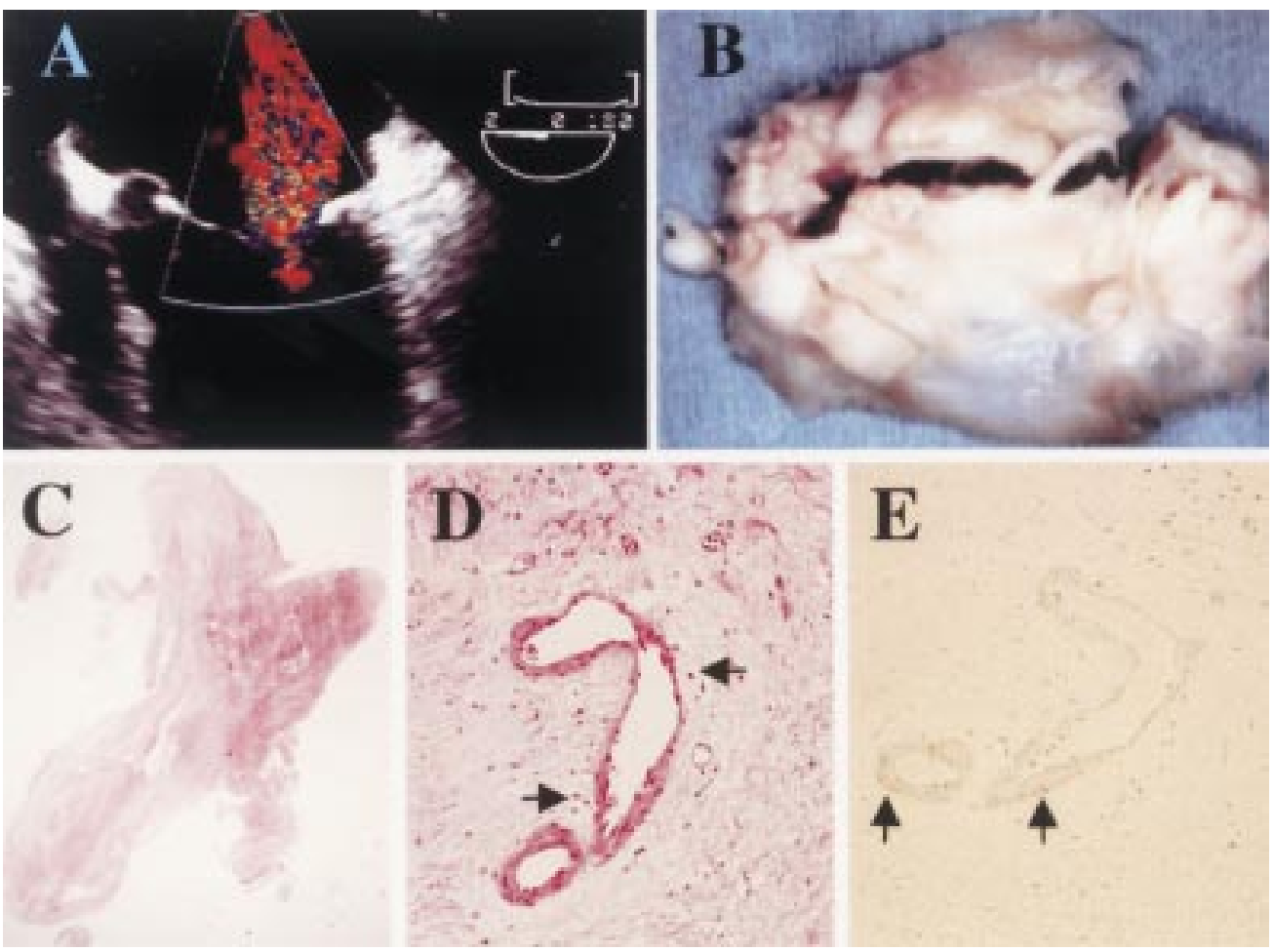

Figure 1 (A) Transoesophageal echocardiograph, revealing severe mitral regurgitation, a thickened mitral leaflet, and a dilated left atrium. (B) Resected mitral valve showing diffuse thickening of both leaflets. (C, D) Haematoxylin and eosin staining $(\times 400)$ revealing perivascular mononuclear infiltration (arrow). (E) Immunostaining using a monoclonal 


\section{Discussion}

Several clinical studies have demonstrated that valvar abnormalities develop in $36 \%$ of patients with primary APS, suggesting that antiphospholipid antibodies (aPL) may play a role in the pathogenesis of valvar lesions. ${ }^{12}$ Pierangeli and colleagues showed the thrombogenicity of aPL by activating endothelial cells in vivo with aPL and correlated these findings with the in vitro expression of adhesion molecules (VCAM-1) on the surface of aPL (APLIgG) - exposed human umbilical vein endothelial cells. ${ }^{3}$ However, in our patient testing for APL-IgG activity was negative, and lupus anticoagulant was the only type of aPL that was detected. In Pierangeli's report, variable stimulation of the expression of VCAM-1 by six preparations of aPL was observed, suggesting a heterogeneity in the function of different aPL.
Furthermore, the concept of heterogeneity between different autoantibodies associated with APS is strengthened by several experimental reports. ${ }^{4}$ Therefore, the valvar lesion in our patient may have been caused by activation of one or more processes by aPL.

We conclude that VCAM-1 expression on the mitral valve may lead to valvar pathology in patients with primary APS.

1 Hoinic M, George J, Ziporen L, et al. Heart valve involvement (Libman-Sacks endocarditis) in the antiphosinvolvement (Libman-Sacks endocarditis) in the
pholipid syndrome. Circulation 1996;93:1579-87.

pholipid syndrome. Circulation 1996;93:1579-87.
2 Brenner B, Blumenfeld Z, Markiewicz W, et al. Cardiac involvement in patients with primary antiphospholipid syndrome. F Am Coll Cardiol 1991;18:931-6.

3 Pierangeli SS, Colden-Stanfield M, Liu X, et al. Antiphospholipid antibodies from antiphospholipid syndrome patients activate endothelial cells in vitro and in vivo. Circulation 1999;99:1997-2002.

4 Greaves $M$. Antiphospholipid antibodies and thrombosis. 